\title{
Benzodiazepine erhöhen Demenzrisiko nicht generell
}

\author{
Benzodiazepine werden häufig gegen Angst, Unruhe und Schlaflosigkeit verordnet. Gerade \\ bei älteren Menschen besteht aber der Verdacht, dass sie möglicherweise eine Demenz \\ fördern oder beschleunigen. Diese Hypothese wurde nun im Rahmen einer prospektiven, \\ populationsbasierten Kohortenstudie geprüft.
}

Ausgewertet wurden die Daten von 3434 Personen über 65 Jahren des GroupHealth-Gesundheitsversorgungs-Systems in Seattle. Darin werden u.a. Medikamenten-Verordnungen computerbasiert erfasst. Eingeschlossen wurden die Teilnehmer in den Zeiträumen 1994-1996, 2000-2003 und seit 2004

Die Probanden wurden zu Beginn und anschließend in zweijährigen Intervallen mit dem Cognitive-abilities-screening-Instrument auf Demenzsymptome untersucht. Bestand der Verdacht auf eine Demenz, wurde dieser durch die neurologischpsychologische Standarddiagnostik überprüft. Um den Benzodiazepin-Konsum zu beurteilen, wurde die „total standardized daily dose“ (TSDD) im vorangegangenen
10-Jahres-Zeitraum berechnet. Das Jahr vor der jeweiligen Erhebung wurde nicht berücksichtigt, um eine mögliche Benzodiazepin-Gabe zur Behandlung von Demenz-Prodromi auszuschließen.

Innerhalb der medianen Nachbeobachtungszeit von 7,3 Jahren entwickelten 23,2\% der Studienteilnehmer eine Demenz. Die adjustierte Hazard Ratio der Teilnehmer, die Benzodiazepine erhalten hatten, betrug im

- niedrigen Dosisbereich

(1-10 TSDDs) 1,25 (95\%-Konfidenz-

intervall 1,03-1,51),

- mittleren Dosisbereich (31-120 TSDDs) 1,31 (1,00-1,71) und

- hohen Dosisbereich

(>121 TSDDs) $1,07(0,82-1,39)$.
Somit war ein höherer BenzodiazepinKonsum nicht generell mit einem höheren Demenz-Risiko vergesellschaftet, sondern nur im niedrigen bis mittleren Dosisbereich. Zwischen Demenz im Allgemeinen und Alzheimer-Demenz gab es keine Unterschiede. Die Autoren raten trotzdem dazu, Benzodiazepine bei älteren Personen zu vermeiden - auch wegen des hohen Gefahrenpotenzials wie Sturz und Abhängigkeit.

Dr. med. Peter Pommer, Oberammergau DOI 10.1055/s-0042-102977

Grey et al. Benzodiazepine use and risk of ... BM] 2016; 352: i90

\section{Kommentar aus der Praxis}

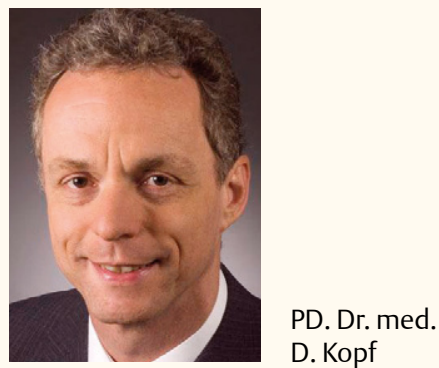

Benzodiazepine beeinträchtigen akut kognitive Leistungen wie Lernen und Gedächtnis - aber begünstigen sie langfristig auch die Entstehung einer Demenz? Die Studie von Gray et al. scheint hier Entwarnung zu geben, nachdem ältere Studien zu dieser Frage uneinheitlich waren.

Die Studie ist sehr sorgfältig konzipiert und besticht durch ein prospektives Design über einen langen Beobachtungszeitraum von bis zu 11 Jahren. Dadurch wird die Wahrscheinlichkeit eines Fehlschlusses reduziert: Benzodiazepine könnten für frühe Begleitsymptome ei- ner Demenz verordnet worden sein. Dadurch könnte eine Assoziation entstehen, die als Kausalität missverstanden werden könnte. Dieser Fall konnte durch das sorgfältige Design umgangen werden.

Komplette Entwarnung lässt sich allerdings noch nicht geben:

- Erstens waren die kumulativen Benzodiazepin-Dosen auch in der Hochdosisgruppe im Median noch mäßig.

- Zweitens ist erst kürzlich eine europäische Studie erschienen, die bei einer ähnlich langen Laufzeit doch eine leichte Erhöhung des Demenzrisikos durch Benzodiazepine findet (Alzheimers Dement 2015; pii: S1552-5260(15)02953-2). Dieser Zusammenhang bestand vor allem für Benzodiazepine mit einer langen Plasmahalbwertszeit.

- Drittens bringt - bei dem sehr langen Vorlauf der Neurodegeneration - auch ein Studienzeitraum von 10 Jahren noch keine absolute Sicherheit.

- Und viertens, das ist der entscheidende Grund, erhöht die dauerhafte Ein- nahme von Benzodiazepinen weitere im Alter entscheidende Risiken wie z.B. das Sturzrisiko oder das Risiko einer Abhängigkeit mit der Gefahr eines Delirs oder Entzugssyndroms beim Absetzen.

Benzodiazepine sind im Rahmen eines umfassenden Behandlungskonzepts für depressive Störungen oder Angststörungen hoch wirksame und essentielle Medikamente. Dabei eignen sie sich insbesondere für die Initialphase der Therapie. Die Indikation für eine langfristige Therapie oder als Bedarfsmedikation ohne klaren Einnahmemodus soll jedoch sehr zurückhaltend und nur nach Ausschöpfen anderer Optionen gestellt werden. Dies gilt auch unabhängig von einem potenziellen Demenzrisiko.

PD. Dr. med. Daniel Kopf Chefarzt der Geriatrischen Klinik, Marienkrankenhaus, Hamburg

Interessenkonflikt

Der Autor gibt an, dass kein Interessenkonflikt besteht. 